\title{
Is acculturation a risk factor for early smoking initiation among Chinese American minors? A comparative perspective
}

\author{
Xinguang Chen, Jennifer B Unger, C Anderson Johnson
}

\begin{abstract}
Objective-To determine the extent to which Chinese American and white minors differ in age of smoking initiation, and to determine the effect of acculturation on smoking initiation.

Design-Cross-sectional telephone surveys.

Setting-Stratified random samples of the state of California, United States.

Subjects-347 Chinese American and 10129 white adolescents aged 12 through 17 years, from the California Tobacco Survey (1990-93) and the California Youth Tobacco Survey (1994-96).

Outcome measures-Hazards (risk) of smoking initiation by age, smoking initiation rate, cumulative smoking rate, mean age of smoking initiation, and acculturation status.
\end{abstract}

Statistical methods-Life table methods, proportional hazards models, and $\chi^{2}$ tests. Results-The risk of smoking initiation by age among Chinese American minors was about a third of that among white minors. The risk for Chinese Americans continued to rise even in later adolescence, in contrast to that for whites, which slowed after 15 years of age. Acculturation was associated significantly with smoking onset among Chinese Americans. Acculturation, smoking among social network members, attitudes toward smoking, and perceived benefits of smoking were associated with the difference in hazards of smoking onset between Chinese American minors and their white counterparts.

Conclusions-Chinese American adolescents had a lower level and a different pattern of smoking onset than white adolescents. Levels of acculturation and other known risk factors were associated with the hazards of smoking initiation among Chinese American minors and with the difference in smoking initiation between the Chinese and white adolescents. Tobacco prevention policies, strategies, and programmes for ethnically diverse populations should take acculturation factors into account. (Tobacco Control 1999;8:402-410)

Keywords: acculturation, Chinese Americans, smoking initiation, adolescence
Introduction

Tobacco control researchers recently have recognised the need to focus prevention efforts on young people in ethnic minorities to curb the increasing trend of tobacco use in the United States. ${ }^{1-6}$ Asian Americans are one of the fastest growing ethnic minority groups in the United States, but smoking among Asian American adolescents has not been studied thoroughly. ${ }^{7}$ Most large-scale studies on smoking issues have included Asian/Pacific Islander Americans in their study samples, but only a few have reported results in detail for Asians in general $^{1}$ and by some subgroups, such as the reports with data from the California Behavioral Risk Factor Surveys for Chinese Americans in $1989,{ }^{7}$ Vietnamese Americans in $1991,{ }^{8}$ and Korean Americans in $1994 .^{9}$

Chinese Americans, as a subgroup of Asian/ Pacific Islander Americans in the United States, show lower rates of cigarette smoking than do many other ethnic groups, including other Asian subgroups as well as non-Asian Americans. ${ }^{5}$ 10-13 Among Chinese American adults, $28 \%$ of men and $1 \%$ of women were current smokers. ${ }^{57}$ These rates are lower than the prevalence rates observed among other ethnic subgroups and the total population in the United States. ${ }^{13-17}$ These rates are also lower than the $67 \%$ and $4 \%$ prevalence rates observed among Chinese adult men and women in the 1996 National Prevalence Survey of Smoking Pattern in China. ${ }^{18}$ Similarly, among Chinese American adolescents, approximately $10 \%$ (about $12-14 \%$ for males and $6-8 \%$ for females) reported having ever smoked cigarettes in their lifetime, lower than the $20-35 \%$ prevalence rate for males and females observed among adolescents from various non-Asian ethnic groups in the United States ${ }^{10}{ }^{11}{ }^{18}$; and about the same as or slightly lower than that of Chinese adolescents in China. ${ }^{19} 20$

Studies have shown that the remarkable ethnic differences in smoking prevalence and initiation in the United States are associated with or mediated through an array of factors, such as genetic differences, socioeconomic status, peer pressure, cultural factors, media influences, and school performance. ${ }^{21-24}$ Among these factors acculturation is one that has received research attention in recent years. ${ }^{25-27}$ There have been debates on the definition of acculturation, the processes by which it occurs, and the measurement of acculturation level. In general, the term 
Table 1 Characteristics of the study samples

\begin{tabular}{|c|c|c|c|c|c|}
\hline & \multicolumn{2}{|c|}{ Chinese American } & \multicolumn{2}{|l|}{ White } & \multirow[b]{2}{*}{$p$} \\
\hline & $n$ & $\%$ & $n$ & $\%$ & \\
\hline \multicolumn{6}{|c|}{ Source/survey year } \\
\hline CTS & 236 & 68.01 & 7139 & 70.48 & \multirow[t]{4}{*}{$>0.05$} \\
\hline 1990-91 & 121 & 34.87 & 2952 & 29.14 & \\
\hline 1992 & 30 & 8.65 & 972 & 9.60 & \\
\hline 1993 & 85 & 24.50 & 3215 & 31.74 & \\
\hline CYTS & 111 & 31.99 & 2990 & 29.56 & \multirow[t]{5}{*}{$>0.05$} \\
\hline 1994 & 29 & 8.36 & 842 & 8.31 & \\
\hline 1995 & 39 & 11.24 & 994 & 9.81 & \\
\hline 1996 & 43 & 12.39 & 1154 & 11.39 & \\
\hline Total & 347 & 131.99 & 10129 & 129.52 & \\
\hline \multicolumn{6}{|l|}{ Sex } \\
\hline \multicolumn{6}{|l|}{ CTS } \\
\hline Male & 119 & 50.42 & 3599 & 50.41 & \multirow[t]{2}{*}{$>0.05$} \\
\hline Female & 117 & 49.58 & 3540 & 49.59 & \\
\hline \multicolumn{6}{|l|}{ CYTS } \\
\hline Male & 59 & 53.15 & 1483 & 49.60 & \multirow[t]{2}{*}{$<0.05$} \\
\hline Female & 52 & 46.85 & 1507 & 50.40 & \\
\hline \multicolumn{6}{|l|}{ Total } \\
\hline Male & 178 & 51.30 & 5082 & 50.17 & \multirow[t]{2}{*}{$>0.05$} \\
\hline Female & 169 & 48.70 & 5047 & 49.83 & \\
\hline \multicolumn{6}{|c|}{ Mean age (years) } \\
\hline CTS & 14.6 & (SD 1.80) & 14.4 & (SD 1.71) & $>0.05$ \\
\hline CYTS & 14.5 & (SD 1.66) & 14.5 & (SD 1.67) & $>0.05$ \\
\hline Total & 14.6 & (SD 1.76) & 14.4 & (SD 1.70) & $>0.05$ \\
\hline
\end{tabular}

CTS = California Tobacco Survey; CYTS = California Youth Tobacco Survey.

acculturation has been defined as the process by which members of a culturally different ethnic group adopt the cultural characteristics, including customs, beliefs, social norms, and values, of another group (the dominant or the mainstream culture) in which they live. ${ }^{328-32} \mathrm{~A}$ series of studies of Latino/Hispanic American minors indicated that the more acculturated subjects experienced a higher level of risk of early smoking initiation and a higher prevalence rate of cigarette smoking than did those who were less acculturated..$^{272-35} \mathrm{~A}$ few studies of Asian Americans also have reported that acculturation may play a role in cigarette smoking. ${ }^{12}{ }^{15}$ As the level of acculturation increases, the rates of smoking initiation, prevalence, and quitting for Asian Americans approach those for whites. However, none of these studies focused on Chinese Americans.

A thorough understanding of the low rate of cigarette smoking among Chinese American adolescents is important not only for effective tobacco control among Chinese Americans in the United States but also for an improved understanding of the aetiology of smoking among people from other ethnic backgrounds. However, little research has been done in this area. It is not clear why Chinese American adolescents show a lower prevalence of cigarette smoking than do all other adolescents in the United States, and even their native counterparts in China. The acculturation process may play a role in determining smoking among Chinese American adolescents, but this association also has not been researched adequately. Assuming that cultural differences in tobacco use between China and the United States affect the smoking behaviour of Chinese American adolescents, this study examines two questions: (a) How and to what extent do Chinese American minors differ from white minors in smoking initiation? (b) Is acculturation a factor contributing to smoking initiation and the differences in smoking initiation between these two ethnic groups?

\section{Method}

DATA SOURCES AND STUDY SUBJECTS

Data used in this study are from the California Tobacco Survey (CTS) conducted by the University of California San Diego in 1990-91, 1992, and 1993, and the California Youth Tobacco Survey (CYTS) conducted by the California Department of Health Services in 1994, 1995, and 1996. Both surveys were parts of the California Tobacco Control Program under the state Proposition 99-the Tobacco Tax and Health Promotion Act of 1988. Both surveys obtained random samples of adolescents in California and used identical, computer-aided telephone interview (CATI) techniques for data collection. Extensive use of these datasets for the monitoring and evaluation of the California Tobacco Control Program indicated that the data from these two sources are reliable. ${ }^{14}{ }^{19}$ 36-38 Previous studies and our analysis in this study have demonstrated that the samples from these two sources are similar in demographic characteristics, except that the CYTS data covered about 6\% more Chinese American boys than girls (table 1). Therefore, the data from the two surveys have been combined for use in this study to increase the number of Chinese American respondents available for data analysis.

Respondents coded as Chinese Americans were used as the primary subjects for the study, and respondents coded as white (non-Hispanic whites) in the same datasets were selected as a reference group representing the mainstream culture for comparison purposes. There are a total of 349 Chinese American subjects in the combined dataset. Among these, two subjects were excluded from the sample because of missing data on most variables such as age, English skills, and language at home. Therefore, 347 Chinese American and 10129 white subjects aged $12-17$ years were included in the statistical analysis (see table 1 for further details of sample characteristics).

MEASUREMENT OF SMOKING INITIATION

Three statistics were used to assess smoking initiation behaviour: rates of smoking initiation, hazards (risk) of smoking initiation by age, and mean age of smoking initiation. First, respondents who responded "yes" to the question "Have you ever smoked a whole cigarette?" were defined as smoking initiators. These smoking initiators then were further asked about the age when they first had smoked a whole cigarette. This reported age was defined as the age of smoking initiation, which was used in subsequent statistical analyses and computations. The self-reported age of smoking initiation could be biased by the age of the subjects at survey time. ${ }^{39}$ To examine this issue, correlation analyses were conducted between the self-reported age of smoking initiation and the age of subjects at survey, yielding an $R^{2}$ of $0.13(\mathrm{p}>0.05)$ for Chinese American minors and $0.12(\mathrm{p}<0.01)$ for white minors. This result indicated that although the 
recalled age of smoking initiation was not statistically independent from the age at survey for white minors, only $12 \%$ of the variances of the recalled age is attributable to the age of the subjects at survey, which is limited.

The smoking initiation rate was defined and computed as a ratio of the number of smoking initiators over the total number of respondents at a given age. A mean age of smoking initiation was defined and computed as the arithmetic mean of the reported age of smoking initiation among the respondents who had smoked at least one whole cigarette. There are studies which have reported that the arithmetic mean age of smoking initiation estimated this way is usually biased downward. ${ }^{14}$ To overcome this bias, an adjusted mean age of smoking initiation was estimated based on a survival model analysis of the smoking initiation process. $^{124041}$

Given the information on smoking status and age of smoking initiation, the hazards of smoking initiation by age were defined and estimated as the instantaneous rate (probability) that a non-smoker at the beginning of a given age may start smoking within that year. These hazards were estimated using life table techniques. ${ }^{41} 42$ Epidemiologically these hazards of smoking initiation measure quantitatively the net risk that a non-smoker may start smoking at any time during a given age period. ${ }^{12}$

Given the estimated hazards of smoking initiation by age, we defined and estimated the cumulative smoking rate by age. This is a ratio of the total number of smoking initiators from birth to a given age over the number of people in a hypothetical population, assuming that this population were exposed continuously to the current social and environmental conditions. The smoking rate estimated this way is not biased by the age structure of a study population; it can be used directly to make comparisons among subgroups. ${ }^{40}$

VARIABLES FOR ACCULTURATION MEASUREMENT Three variables were selected to measure acculturation status: (a) English skills measured on a five-point scale; (b) language spoken at home, also measured on a five-point scale; and (c) age (in years) when first entering the United States. In the original datasets, language spoken was recorded as $1=$ only native language spoken, $2=$ native language spoken more than English, 3 = both spoken equally, 4 $=$ English spoken more than native language, and $5=$ only English spoken. Because approximately $70 \%$ of the Chinese American minors reported speaking only English at home and using only English for communications, these two language variables were re-coded as dichotomous variables, with $1=$ only English spoken and $0=$ any use of native languages.

The age at first arrival in the United States was a continuous variable in the original datasets. This information was obtained from responses to the question: "What was your age when you first entered the United States?" The distribution of this variable was extremely skewed: $68 \%$ of the respondents reported that they had been born in the United States, and $0.5-2 \%$ of the respondents reported that they had arrived in the United States between one and 16 years of age. This variable was therefore re-coded as a dichotomous variable, where $1=$ born in the United States or arrived at or before six years of age, and $0=$ entered the United States after their seventh birthday, assuming that acculturation levels of the Chinese who immigrate to the United States before entering school do not differ significantly from the second-generation Chinese Americans in the United States by adolescence.

It would have been better to have had a full measurement of acculturation level, but this requires other variables such as selfidentification of ethnicity and cultural assimilation, in addition to language use and years of residence in the United States. These additional variables were not available in the datasets used in this analysis. However, the three variables we selected are common and are fundamental components of acculturation scales used in many documented epidemiological and behavioural studies. ${ }^{32-35}$ 43-46 $^{4}$ Furthermore, even language variables have been shown to be reliable and valid acculturation measures when used alone..$^{27} 344345$

\section{COVARIATES}

To detect associations between acculturation and smoking initiation among Chinese American minors, other variables known to be associated with cigarette smoking and smoking initiation were included in the statistical models as control variables (covariates). Any observed associations between acculturation and smoking might be partially or fully confounded by or mediated through these variables. For example, peer pressure, socioeconomic status, and depression have been shown to be associated with cigarette smoking and acculturation. ${ }^{25-27} 3243$

After a review of the research literature and an examination of the variables available in the datasets, 16 variables in six categories were selected as control variables.

- Demographic variables: age (in years); sex ( 1 = male, 2 = female); year of survey (chronological year)

- Variables of social network smoking status: reported smoking status of peers, siblings, parents, and teachers $(1=$ smoker, $0=$ nonsmoker)

- Tobacco media exposure $(1=$ exposed, $0=$ not exposed)

- Attitudes toward cigarette smoking: if hate being around people who are smoking; if agree that smoking turns me off; if feel OK when around smokers $(1=$ yes, $0=$ no $)$

- Perceived benefits of smoking: if agree that smoking is good at parties; if agree that smoking aids in relaxation; and if agree that smoking alleviates boredom $(1=$ yes, $0=$ no)

- Perceived parental approval of smoking: if think that parents will approve of my smoking when I grow up ( $1=$ yes, $0=$ no). 
STATISTICAL METHODS AND DATA ANALYSIS Statistical analysis was completed using the SAS system version $6.12 .{ }^{47}$ All variables used in this study were re-coded according to the definitions specified in this study before statistical analysis and computation. Data also were examined for any missing entries and any possible logical errors before analysis.

In addition to descriptive statistics such as means, proportions, and rates, survival models ${ }^{39-41}$ were used to estimate the hazards of smoking initiation and the cumulative smoking rate. In the survival models, the age when a person first smoked a whole cigarette was defined as the "survival" time. All those who reported having smoked a whole cigarette were treated as "failure" events; and the rest who did not report smoking initiation before the time of the survey were treated as censored, according to the methods of survival analysis. ${ }^{40} 41$

There are several advantages to using survival models to examine age of smoking initiation. First, they can produce better estimations of age of smoking initiation than other methods. With cross-sectional, multiage-group data, age of smoking initiation cannot be estimated correctly because, for example, a subject aged 12 at the time of the survey cannot report a possible age of smoking initiation older than 12. Survival models can overcome this problem. ${ }^{12}$ Second, when using survival models, not only the mean age of smoking initiation can be estimated, but also a probability curve of smoking initiation by age can be obtained with cross-sectional data. This is usually only obtainable through data collected in expensive and time-consuming longitudinal studies. ${ }^{40}$

SAS PROC LIFETEST with the option of ACT (actuarial) ${ }^{47}$ was used to estimate the hazards of smoking initiation. This computation procedure also produced a set of other results in addition to the hazards of smoking initiation using the life table method. The logrank test was used to compare the estimated age trend of hazards of smoking initiation between Chinese American and white minors. $\chi^{2}$ and $t$ tests were used as bivariate methods to assess associations between risk/protective factors and smoking initiation. SAS PROC PHREG (proportional hazards regression model) with an option of backward

Table 2 Differences in smoking initiation between Chinese American and white subjects. Adolescents 12-17 years old, male and female, California, 1990-1996

\begin{tabular}{lllll}
\hline Smoking measure & Sex & $\begin{array}{c}\text { Chinese } \\
\text { American } \\
(n=347)\end{array}$ & $\begin{array}{l}\text { White } \\
(n=10129)\end{array}$ & $p$ \\
\hline Rate of smoking initiation (\%) & Boys & 13.5 & 29.8 & $<0.01$ \\
& Girls & 8.3 & 28.5 & $<0.01$ \\
& Total & 10.9 & 29.2 & $<0.01$ \\
Mean age of smoking initiation (years) & Boys & 12.8 & 12.5 & $>0.05$ \\
& Girls & 13.1 & 13.0 & $>0.05$ \\
& Total & 12.9 & 12.7 & $>0.05$ \\
Adjusted mean age of initiation (years) & Boys & 14.8 & 14.6 & $>0.05$ \\
& Girls & 14.4 & 15.0 & $>0.05$ \\
& Total & 14.6 & 14.9 & $>0.05$ \\
Cumulative smoking rate at age 15-17 (\%) & Boys & 17.2 & 42.6 & $<0.01$ \\
& Girls & 13.8 & 43.0 & $<0.01$ \\
& Total & 16.1 & 42.8 & $<0.01$ \\
Self-reported 30-day smoking rate (\%) & Boys & 3.4 & 11.7 & $<0.01$ \\
& Girls & 1.2 & 12.3 & $<0.01$ \\
& Total & 2.3 & 11.9 & $<0.01$ \\
\hline
\end{tabular}

selection $^{40} 4148$ was used as a multivariate method to examine the net effect of the acculturation variables on the hazards of smoking initiation while controlling for all potential confounding variables selected.

\section{Results}

Among 10476 subjects in the analytical dataset, $347(3.3 \%)$ were Chinese Americans and $10129(96.7 \%)$ were whites. Among the 347 Chinese American subjects, 51.3\% were male; of the 10129 white subjects, $50.2 \%$ were male, giving a total of 5260 males $(50.2 \%)$. The mean age was 14.6 (SD 1.76) for Chinese and 14.4 (SD 1.70) for whites. About $70 \%$ of the subjects were questioned in the California Tobacco Survey in 1990-91, 1992, and 1993; and about $30 \%$ were interviewed in the California Youth Tobacco Survey in 1993, 1994, 1995, and 1996. There were no significant differences between Chinese American subjects and white subjects in their mean age, sex ratio, and proportion distribution by source and year of survey at the $\mathrm{p}<0.05$ level, except that the CYTS data covered more male subjects than females for Chinese Americans (table 1).

Most $(89.6 \%)$ of the Chinese American minors reported that they had first arrived in the United States at age six or younger, or had been born in the United States. Slightly over $10 \%$ of the Chinese American minors reported having immigrated to the United States at seven or older. About $70 \%$ of the Chinese American minors reported speaking only English at home and using only English for communication, whereas $98-99 \%$ of white minors reported speaking only English at home and using only English for communication.

The estimated smoking initiation rates were $13.5 \%$ and $8.3 \%$ for Chinese American boys and girls respectively, lower than the rates for white boys and girls $(29.8 \%$ and $28.5 \%$, $\mathrm{p}<0.01)$. The estimated cumulative smoking initiation rates by age 17 were $17.2 \%$ and $13.8 \%$ for Chinese American boys and girls respectively, and $42.6 \%$ and $43.0 \%$ for white boys and girls. These differences were also significant $(\mathrm{p}<0.01)$. The estimated arithmetic mean age of smoking onset was 12.8 years of age and 13.1 years of age for Chinese American boys and girls respectively. These are slightly older than the ages of 12.5 and 13.0 for their white counterparts, but not significantly different $(p>0.05)$. The adjusted mean age of smoking onset was 14.8 and 14.4 years of age for Chinese American boys and girls, and 14.6 and 15.0 for their white counterparts. There are approximately two-year differences between the arithmetic and adjusted mean age of smoking initiation for both groups (table 2).

Figure 1 presents the estimated hazards of smoking initiation by age for Chinese American and white adolescents. First, the graph shows a clear and similar age trend of hazards of smoking initiation for these two groups of adolescents by gender. Before 10 years of age, the hazards curves of smoking initiation for both Chinese American and white minors were rather low, less than $2 \%$ each year. 


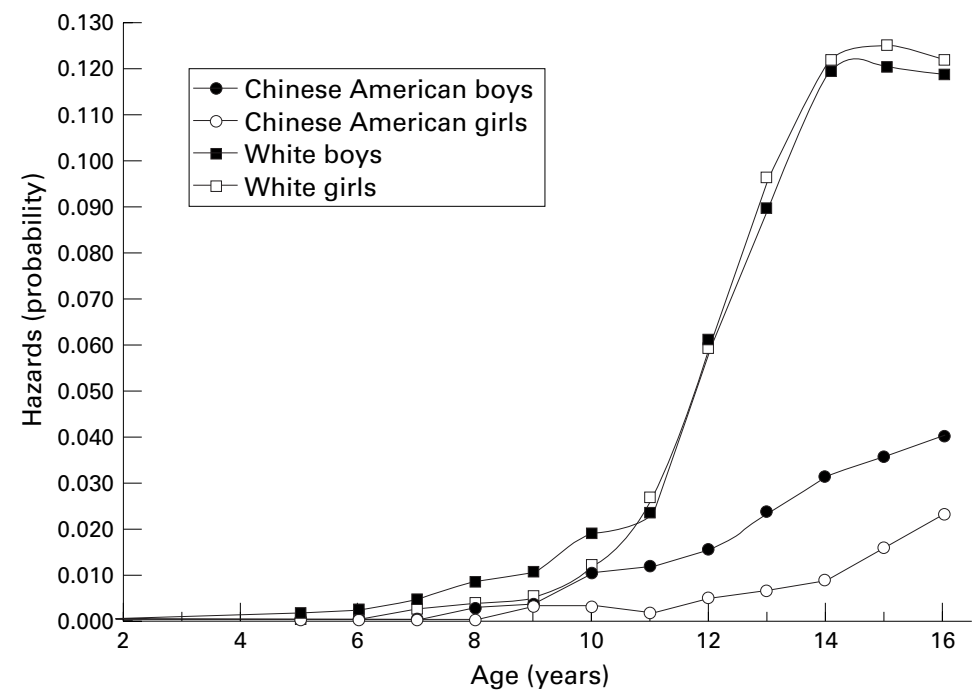

Figure 1 Comparison of hazards of smoking initiation between Chinese American minors and their white counterparts, 1990-96, California.

This means that, of 100 non-smoking adolescents under 10 years of age, approximately two would be expected to try smoking each year. After 10 years of age, however, the hazards curves for Chinese Americans and whites increase sharply, indicating a rapid increase in the risk of smoking initiation with age among both groups. However, the speed of increase is slower for Chinese American than for white adolescents.

Second, the hazards curve was higher for boys than for girls among Chinese Americans $(p<0.05)$, but not among whites $(p>0.05)$

Third, the hazards of smoking onset for Chinese Americans, although only about a third of that of their white counterparts, shows a monotonic increase that continues through the oldest age group (16-17 years). Among whites, however, the increasing risk of smoking initiation began to slow after $14-15$ years of age. Log-rank tests indicated that the differences in age trend of the hazards of smoking initiation between Chinese American minors and whites for either boys or girls was extremely significant $(\mathrm{p}<0.01)$.

Table 3 Comparison of percentage differences in factors known to be associated with cigarette smoking between Chinese American minors and their white counterparts

\begin{tabular}{|c|c|c|c|c|c|}
\hline Factors & Chinese & White & Ratio & $\chi^{2}$ & $p$ \\
\hline \multicolumn{6}{|l|}{ Social network smoking } \\
\hline Parent smoking & 20.58 & 33.54 & 0.61 & 25.29 & 0.001 \\
\hline Sibling smoking & 4.64 & 12.09 & 0.38 & 17.74 & 0.001 \\
\hline Friend smoking $(\mathrm{m})$ & 19.52 & 35.92 & 0.54 & 33.31 & 0.001 \\
\hline Friend smoking (f) & 13.16 & 31.46 & 0.42 & 46.22 & 0.001 \\
\hline Teacher smoking & 74.92 & 82.06 & 0.91 & 10.32 & 0.001 \\
\hline \multicolumn{6}{|l|}{ Media exposure to tobacco } \\
\hline Exposed to media & 45.53 & 51.92 & 0.88 & 9.49 & 0.002 \\
\hline \multicolumn{6}{|c|}{ Attitude towards smoking behaviour } \\
\hline Hate being around smokers & 83.57 & 72.74 & 1.15 & 20.02 & 0.001 \\
\hline Smokers turn me off & 81.84 & 80.70 & 1.01 & 0.28 & 0.595 \\
\hline Feel OK around smokers & 19.02 & 30.90 & 0.62 & 22.34 & 0.001 \\
\hline \multicolumn{6}{|c|}{ Perceived benefits of cigarette smoking } \\
\hline Good for party & 40.06 & 45.60 & 0.88 & 4.16 & 0.041 \\
\hline Helps relax & 37.75 & 34.53 & 1.09 & 1.53 & 0.215 \\
\hline Relieves boredom & 19.88 & 17.58 & 1.13 & 1.22 & 0.269 \\
\hline \multicolumn{6}{|c|}{ Perceived parents' approval of smoking } \\
\hline Parent OK with my smoking & 11.53 & 15.41 & 0.75 & 3.91 & 0.048 \\
\hline \multicolumn{6}{|l|}{ Acculturation variables } \\
\hline Age in US $>6$ years & 89.63 & 99.25 & 0.90 & 293.82 & 0.0001 \\
\hline Language at home & 70.14 & 98.70 & 0.71 & 1238.62 & 0.0001 \\
\hline English skills & 69.45 & 98.44 & 0.71 & 1146.41 & 0.0001 \\
\hline
\end{tabular}

Table 3 compares the differences between Chinese American and white adolescents in the distribution of the 16 selected covariates and three acculturation variables. This table shows that Chinese American minors were exposed substantially less than their white counterparts to smoking among social network members such as friends and siblings. The levels of these variables for Chinese Americans ranged from $38 \%$ to $91 \%$ of those of the white subjects, and all the differences were highly significant at $p<0.001$. For example, about $32 \%$ of the white minors reported that their best female friends were smokers, whereas only $13 \%$ of the Chinese American minors reported the same.

Compared with white adolescents, fewer Chinese Americans reported exposure to pro-tobacco media and parental approval of smoking. Chinese American adolescents were more likely than were white adolescents to report negative attitudes toward other people's smoking $(40 \% v 46 \%, \mathrm{p}<0.05)$.

BIVARIATE ANALYSES

To examine differences in smoking behaviours between Chinese American and white adolescents, we tested associations between all the selected variables and cigarette smoking initiation for Chinese Americans and whites separately (table 4). Among the five social network smoking variables, influence of smoking parents on teenagers' smoking initiation is greater for Chinese Americans (relative risk, $R R=3.01)$ than for whites $(R R=1.68)$. The influences of the other four variables in this category (smoking among siblings, male and female friends, and teachers) are weaker for Chinese American minors than for white minors. For tobacco media exposure, no significant association with smoking initiation was found for Chinese American minors, and an RR of 1.4 was observed for the white minors $(\mathrm{p}<0.001)$. The protective effect of negative attitudes towards cigarette smoking was slightly weaker for Chinese Americans than for whites. For example, the relative risk for smoking initiation among subjects who disliked being around smokers was 0.13 for Chinese American subjects, and 0.17 for their white counterparts. Supportive attitude toward smoking was a weaker risk factor for smoking initiation among Chinese Americans ( $R R=$ 3.94) than among whites $(R R=5.49)$.

Two of the three variables of perceived benefits from smoking (smoking aids in relaxation, and smoking helps relieve boredom) were associated with an increased risk of smoking initiation for both Chinese American and white minors. The effect for Chinese Americans was not as strong as for whites. The relative risk of smoking initiation for those who believed smoking aids in relaxation was $1.18(\mathrm{p}<0.05)$ for Chinese Americans and $1.90(p<0.01)$ for whites. The same risk for those who believe smoking helps relieve boredom was 2.39 $(\mathrm{p}<0.01)$ for Chinese Americans and 2.90 $(\mathrm{p}<0.01)$ for whites. The belief that smoking helps one to have fun at parties increased the risk of smoking initiation among whites $(R R=$ 
Table 4 Comparison of factors associated with smoking initiation between Chinese American and white adolescents: bivariate analyses

\begin{tabular}{|c|c|c|c|c|c|c|}
\hline & \multicolumn{3}{|c|}{ Chinese Americans } & \multicolumn{3}{|l|}{ Whites } \\
\hline & $\chi^{2}$ & $\begin{array}{l}\text { Relative } \\
\text { risk }\end{array}$ & $p$ & $\chi^{2}$ & $\begin{array}{l}\text { Relative } \\
\text { risk }\end{array}$ & $p$ \\
\hline \multicolumn{7}{|l|}{ Social network smoking } \\
\hline Parent smoking & 8.670 & 3.01 & 0.003 & 125.909 & 1.68 & 0.001 \\
\hline Sibling smoking & 0.253 & 1.48 & 0.615 & 334.594 & 3.02 & 0.001 \\
\hline Friend smoking $(\mathrm{m})$ & 2.438 & 2.32 & 0.118 & 202.552 & 2.92 & 0.001 \\
\hline Friend smoking $(\mathrm{f})$ & 8.129 & 3.03 & 0.004 & 1404.937 & 5.90 & 0.001 \\
\hline Teacher smoking & 7.717 & 3.42 & 0.005 & 1389.325 & 5.85 & 0.001 \\
\hline \multicolumn{7}{|l|}{ Media exposure to tobacco } \\
\hline Exposed to media & 0.519 & 0.76 & 0.471 & 56.359 & 1.40 & 0.001 \\
\hline \multicolumn{7}{|l|}{ Attitude toward smoking behaviour } \\
\hline Hate being around smokers & 23.806 & 0.17 & 0.001 & 1776.429 & 0.13 & 0.001 \\
\hline Smokers turn me off & 2.358 & 0.53 & 0.125 & 1192.294 & 0.18 & 0.001 \\
\hline Feel OK around smokers & 13.997 & 3.94 & 0.001 & 1362.185 & 5.49 & 0.001 \\
\hline \multicolumn{7}{|c|}{ Perceived benefits of cigarette smoking } \\
\hline Good at parties & 1.207 & 0.54 & 0.272 & 316.763 & 2.58 & 0.001 \\
\hline Helps relax & 0.200 & 1.18 & 0.655 & 204.53 & 1.90 & 0.001 \\
\hline Alleviates boredom & 3.701 & 2.39 & 0.054 & 374.193 & 2.90 & 0.001 \\
\hline \multicolumn{7}{|c|}{ Perceived parent's approval of smoking } \\
\hline Parent's approve of my smoking & 3.545 & 2.00 & 0.060 & 593.765 & 3.03 & 0.001 \\
\hline \multicolumn{7}{|l|}{ Acculturation variables } \\
\hline Age in US $>6$ & 0.038 & 0.88 & 0.846 & 0.151 & 0.90 & 0.690 \\
\hline Only English at home & 0.098 & 1.14 & 0.775 & 1.446 & 1.26 & 0.209 \\
\hline English skills & 0.984 & 0.68 & 0.321 & 2.908 & 1.45 & 0.088 \\
\hline
\end{tabular}

2.58, p<0.001), but it was not significant among Chinese Americans $(\mathrm{RR}=0.54, \mathrm{p}=$ 0.272).

Perceived parental approval of smoking was a significant risk factor for smoking initiation for Chinese American and white minors, but the association was weaker for Chinese Americans $(R R=2.00, p<0.01)$ than for whites $(R R$ $=3.03, \mathrm{p}<0.01)$.

Contrary to our expectations, none of the three acculturation variables were associated significantly with smoking initiation among Chinese American or white adolescents in the bivariate analyses.

MULTIVARIATE ANALYSES

Table 5 presents the results of the proportional hazards model analyses. According to the statistic of $-2 \log (\mathrm{L})$, the data fit the model

Table 5 Variables associated with early smoking initiation from proportional hazards model analysis for Chinese American and white adolescents

\begin{tabular}{|c|c|c|c|c|c|c|}
\hline \multirow[b]{2}{*}{ Variables } & \multicolumn{2}{|l|}{ Chinese } & \multicolumn{2}{|l|}{ White } & \multicolumn{2}{|c|}{ Chinese + white } \\
\hline & $R R$ & $p$ & $R R$ & $p$ & $R R$ & $p$ \\
\hline \multicolumn{7}{|l|}{ Social network smoking } \\
\hline Parent smoking & 2.81 & 0.0636 & 1.11 & 0.0184 & 1.11 & 0.0150 \\
\hline Sibling smoking & & & 1.41 & 0.0001 & 1.41 & 0.0010 \\
\hline Best friend smoking $(\mathrm{m})$ & & & 1.59 & 0.0001 & 1.60 & 0.0010 \\
\hline Best friend smoking ( $\mathrm{f}$ ) & & & 1.63 & 0.0001 & 1.64 & 0.0010 \\
\hline Teacher smoking & & & 1.32 & 0.0004 & 1.32 & 0.0010 \\
\hline \multicolumn{7}{|l|}{ Media exposure } \\
\hline Tobacco media exposure & & & 1.47 & 0.0001 & 1.47 & 0.0010 \\
\hline \multicolumn{7}{|l|}{ Attitudes toward smoking } \\
\hline Hates being around smokers & 0.41 & 0.1493 & 0.61 & 0.0001 & 0.61 & 0.0010 \\
\hline Smokers turn me off & & & 0.70 & 0.0001 & 0.70 & 0.0010 \\
\hline Feel OK around smokers & 2.72 & 0.0734 & 1.37 & 0.0001 & 1.37 & 0.0010 \\
\hline \multicolumn{7}{|l|}{ Perceived benefit of smoking } \\
\hline Smoking helps relax & & & 1.24 & 0.0001 & 1.24 & 0.0010 \\
\hline Smoking alleviates boredom & & & 1.16 & 0.0020 & 1.16 & 0.0010 \\
\hline \multicolumn{7}{|c|}{ Perceived parental influence on smoking } \\
\hline Parent's approval of my smoking & & & 1.20 & 0.0003 & 1.20 & 0.0010 \\
\hline \multicolumn{7}{|l|}{ Acculturation status } \\
\hline Only English at home & & & & & 1.77 & 0.0290 \\
\hline Age in US > 6 year & 0.88 & 0.1062 & 1.73 & 0.0295 & 0.95 & 0.0320 \\
\hline \multicolumn{7}{|l|}{ Model test } \\
\hline$-2 \log (\mathrm{L})$ & 165.236 & & 39350.04 & & 39765.12 & \\
\hline DF & 5 & & 15 & & 16 & \\
\hline $\mathrm{p}$ & 0.0007 & & 0.0001 & & 0.0001 & \\
\hline
\end{tabular}

The result is controlled for age, sex, and year of survey.

Due to the relatively smaller sample size, a criteria of $p=0.15$ was used for backward selection in the proportional hazards model analysis for Chinese Americans. quite well for Chinese American subjects and white subjects separately and together $(p<0.001)$. For Chinese American minors, after controlling for other variables, only four variables were associated significantly with early smoking initiation: one social network variable (parents' smoking), one anti-smoking attitude (not liking to be around smokers), one pro-smoking attitude (not minding being around smokers), and one acculturation variable (age of first arrival in the United States). Parents' smoking showed the highest risk $(R R=2.81)$, and the anti-smoking attitude showed the greatest protective effect $(\mathrm{RR}=$ 0.41). Chinese American adolescents who first came to the United States at seven years of age or older had a risk of about $88 \%$ of those who were born in the United States or immigrated to the United States at six or younger.

Among white minors, the age of first arrival in the United States became a significant risk factor $(R R=1.73, p<0.05)$ for early smoking initiation after controlling for the other variables in the model. This indicates that the white adolescents who moved to the United States at seven or older had a $73 \%$ greater probability of early smoking initiation than did those who were born in the United States or moved to the United States at or before six years of age. However, when data from Chinese Americans and whites were pooled together (to reflect the fact that Chinese American and white adolescents were drawn from the same population), the age of first arrival in the United States remained a significant predictor of smoking initiation. The exclusive use of English at home was also a risk factor for cigarette smoking initiation among Chinese Americans and whites $(\mathrm{RR}=1.77, \mathrm{p}<0.05)$. Adolescents who spoke only English at home had a risk of early smoking initiation, 1.77 times greater than that of adolescents who use another language exclusively or partially at home.

\section{Discussion}

The hazards of smoking initiation for Chinese American minors showed a trend similar to the hazards observed among white adolescents; the risk was low before seven years of age and it begins to increase after 10 years of age. This trend of increasing risk during adolescence has been observed in other studies. ${ }^{49}{ }^{50}$ However, throughout early adolescence, the risk of smoking initiation among Chinese American minors is about a third of the risk among whites. Interestingly, although the risk of smoking initiation is lower for Chinese Americans early in adolescence, it continues to increase throughout adolescence, in contrast with the risk among whites, which plateaus in mid-adolescence. This indicates a high risk of smoking initiation in later adolescence and early adulthood for Chinese Americans. Although smoking initiation is rare among Chinese Americans early in adolescence, more may begin smoking later, in mid-to-late adolescence and early adulthood.

Acculturation is an important predictor of early smoking initiation for Chinese American minors. Although the bivariate analysis did not 
show any significant associations between acculturation and early initiation of cigarette smoking, the proportional hazards model analysis indicated that the age of first arrival in the United States was associated significantly with the hazards of smoking initiation by age. Those who came to the United States older than six had a lower risk of smoking initiation than those who arrived at a younger age, or who were born in the United States.

Use of English at home was also associated with early smoking initiation among Chinese Americans. Acculturation may have contributed partially to the Chinese American/white differences in the early initiation of cigarette smoking. The age of first arrival in the United States has opposite effects on smoking initiation for Chinese American and white adolescents. Acculturation increases the risk of smoking initiation among Chinese American minors, but there are relatively more Chinese American minors than white minors in the early stages of acculturation. Acculturation decreases the risk of smoking initiation among white minors, but there are relatively fewer whites than Chinese Americans in the early stages of acculturation.

The results of this study suggest that low acculturation level is not the only variable that protects Chinese American adolescents against early smoking initiation. Only about $30 \%$ of the Chinese American adolescents reported speaking a language other than English at home. Therefore, if acculturation is the only variable affecting smoking initiation among Chinese American adolescents, the remaining $70 \%$ would be expected to have a risk of smoking initiation similar to that of white adolescents. However, this was not the case. Even if the risk of smoking initiation of the unacculturated Chinese Americans were increased to that of the acculturated Chinese Americans, the risk of smoking initiation still would be considerably lower among Chinese Americans than among whites.

This indicates that other variables must account for the observed difference in risk of smoking initiation between Chinese Americans and whites. The results of this study showed that nearly all of the commonly studied risk factors, such as smoking by social network members, pro-tobacco media exposure, and attitudes towards smoking, had weaker effects on Chinese American adolescents than on white adolescents. Therefore, a greater proportion of the variance in smoking initiation remains unexplained among the Chinese Americans. The only exception was parent smoking, which had a stronger association with smoking initiation among Chinese Americans than among whites. This finding is consistent with other studies, ${ }^{11}{ }^{36}$ and it suggests that family factors may be more important in determining smoking behaviour among Chinese American adolescents than among white adolescents.

Other cultural factors, such as the retention of some components of traditional Chinese culture, may also have contributed to the remarkable differences in smoking initiation between Chinese American and whites. Unfortunately, the datasets used in this study do not contain information for examining the issues. Documented studies suggest that the traditional Chinese culture encourages adult men to smoke but strongly discourages women and children from smoking. ${ }^{12}{ }^{51}$ In China, cigarette smoking has usually been treated as a culturally proper behaviour and a social lubricant. For example, it is customary to precede a discussion by offering a cigarette. Many Chinese people have labelled having a cigarette after a meal as having a life "superior to that of God". The smoking rate is much higher among Chinese adolescent and adult males than among females. These tobacco-related cultural elements may influence the smoking behaviour of Chinese Americans, even after they have moved to the United States. These cultural influences may continue to influence Chinese Americans for several generations, discouraging adolescents from smoking but encouraging adult men to smoke. This may explain the existence of gender differences in cigarette smoking among Chinese American adolescents, although these differences are much smaller than that among Chinese in China.

The findings from this study have several implications for smoking prevention policies, strategies, and programmes. We demonstrated that the risk of smoking initiation among Chinese American adolescents monotonically increased throughout mid and late adolescence, and it may continue to increase throughout early adulthood. Therefore, the timing of smoking prevention programmes should be adjusted accordingly for Chinese Americans. Instead of focusing only on middle-school students (ages 11-14), prevention programmes should be extended to high school (ages 15-18) and college $(\geqslant 18)$, to coincide with the period of maximum risk among Chinese Americans. Although research suggests that large-scale smoking prevention programmes are effective among minority adolescents as well as among white minors, ${ }^{42-55}$ the results of this study suggest that the risk of smoking among Chinese Americans may increase later in adolescence, after traditional, school-based smoking prevention programmes have ended.

In addition to the timing of tobacco control, acculturation status should be considered when designing and delivering tobacco control programmes for Chinese American adolescents. The National Cancer Institute's model for community tobacco control recommends that significant subpopulations be targeted with culturally appropriate programming. ${ }^{56}$ The California Tobacco Education and Control Program delivers culturally specific elements for defined target audiences, including the four major ethnic/racial groups in California (African Americans, Asians/Pacific Islanders, Hispanics/Latinos, and Native Americans). ${ }^{7}$ However, no specific tobacco control programme has been developed to target Chinese Americans. Tobacco control policies and interventions are needed to prevent smoking initiation among Chinese American adolescents as they adjust to 
American culture and become more susceptible to pro-tobacco influences from peers and from the media.

There are several limitations to this study. Additional acculturation variables would have allowed for a more complete examination of the role of acculturation in the risk of smoking initiation among Chinese Americans. The variables used in this study can be used to assess the extent to which Chinese Americans have adopted the English language, but they do not indicate whether the Chinese Americans have retained the Chinese cultural values that discourage minors from smoking. According to acculturation theory, the retention of the native culture is an important part of the acculturation process. ${ }^{1127}$ Bicultural adolescents (those who manage to adapt to the American culture while still retaining adaptive aspects of the Chinese culture) may be able to draw from the strengths of two cultures, and may therefore be especially well prepared to resist negative influences on their health.

Use of self-reported age of onset from a cross-sectional data is another limitation. Longitudinal studies demonstrated that older adolescents tend to report a later age of smoking onset than younger ones. ${ }^{39}$ Our analyses indicate that, for white minors, there is a significant correlation between the selfreported age of smoking onset and the age of the subjects at survey. If the sample sizes for Chinese Americans are sufficiently large, the same correlation may also be statistically significant. This age-related recall error, although rather small, will result in certain bias in the estimation of the hazards curve, as well as the associations between the predictor variables and the hazards of smoking onset.

The relatively small number of Chinese American subjects in the study sample is another limitation. Although this study combined data from several years of large-scale surveys, the number of Chinese American adolescents available was still low.

Despite these limitations, this study contributes to the body of knowledge about smoking among Chinese American adolescents. This information about the later risk of smoking initiation among this group, as well as the factors associated with early smoking initiation, can be used to develop more effective and culturally appropriate smoking prevention programmes for Chinese American adolescents.

This study was supported by the University of California Tobacco-Related Disease Research grant 6KT-0191.

1 US Department of Health and Human Services. Tobacco use among U.S. racial/ethnic minority groups: African Americans,
American Indians and Alaska Natives, Asian Americans and American Indians and Alaska Natives, Asian Americans and Pacific Islanders, Hispanics. A report of the Surgeon General,
1998. Atlanta, Georgia: Centers for Disease Control and 1998. Atlanta, Georgia: Centers for Disease Control and
Prevention, Office on Smoking and Health, 1998. (US Prevention, Office on Smoking and Health, 1998. (US
Government Printing Office Publication No S/N 017-001Government-

2 Elder JP, Edward CC, Conway T, et al. Independent evaluation of the California tobacco education program. Public Health Rep 1996;3:353-8.

3 Molina CW, Aguirre-Monila M. Latino health in the US: a growing challenge. Washington DC: American Public growing challenge. Washington

4 Botvin GL, Schinke SP, Epstein JA, et al. Effectiveness of culturally focused and generic skills training approaches to alcohol and drug abuse prevention among minority youths. Psychol Addict Behav 1994;8:116-27.
5 US Centers for Disease Control and Prevention. Cigarette smoking among Chinese, Vietnamese, and HispanicsCalifornia, 1989-91. MMWR 1992;41:362-7.

6 Bal DG, Kizer KW, Felten PB, et al. Reducing tobacco consumption in California: development of a statewide anti-tobacco use campaign. $\mathscr{F} A M A$ 1990;264:1570-4.

7 US Centers for Disease Control and Prevention. Behavioral Risk Factor Survey of Chinese: California 1989. MMWR 1992;41:266-70.

8 US Centers for Disease Control and Prevention. Behavioral Risk Factor Survey of Vietnamese: California 1991. MMWR 1992;41:69-71.

9 US Centers for Disease Control and Prevention. Behavioral Risk Factor Survey of Korean Americans: Alameda Risk Factor Survey of Korean Americans: A
County, California 1994. MMWR 1997;46:774-7.

10 Chen X, Unger JB, Cruz TB, et al. Smoking patterns of Asian American youth in California and their relationships to acculturation. F Adolesc Health 1999;24:321-8.

11 Zane NW, Takeuchi DT, Yang KN, eds. Confronting critical health issues of Asian and Pacific Islander Americans. Thousand Oaks, California: Sage, 1994.

12 Chen X, Unger JB. Hazards of smoking initiation among Asian American and non-Asian adolescents in California: a survival model analysis. Prev Med 1999;28:589-99.

13 Wiecha JM. 1996. Differences in patterns of tobacco use in Vietnamese, African American, Hispanic, and Caucasian Adolescents in Worcester, Massachusetts. Am F Prev Med 1996;12:29-37.

14 US Centers for Disease Control and Prevention. Surveillance for selected tobacco-use behaviors-United States, 1900-1994. MMWR 1997;43(SS-3):2-32.

15 Moeschberger ML, Anderson J, et al. Multivariate profile of smoking in Southeast Asian men: a biochemically verified analysis. Prev Med 1997;26:53-8

6 Gillmore MR, Catalano RF, Morrison DM, et al. Racial differences in accessibility and availability of drugs and early initiation of substance use. Am $\mathcal{F}$ Drug Alcohol Abuse 1990; 16:185-206.

17 Sasao T. Patterns of substance use and health practices among Japanese Americans in Southern California. Paper presented at the Third Annual Meeting of the Asian American Psychological Association, New Orleans, August, 1989 .

18 Yang GH, Fan LX, Tan J, et al. 1996 China National Prevalence Survey of smoking patterns. Beijing: Scientific and lence Survey of smoking patterns. Beijing: Scientific and

19 Li X, Fang X, Stanton B. Cigarette smoking among Chinese adolescents and its association with demographic characteristics, social activities, and problem behaviors. Substance Use Misuse 1996;31:545-63.

20 Chen L, Yu S, Wang Z. Cigarette smoking among middle school students in Jian-Han District, Wuhan, China. 7 Tongij Med Univ Soc Sci Ed 1994;27:29-31.

21 Heath AC, Cates R, Martin NG, et al. Genetic contribution to risk of smoking initiation: comparison across birth cohorts and across cultures. F Substance Abuse 1993; 5:221-46.

22 Escobedo LG, Anda RF, Smith PF, et al. Sociodemographic characteristics of cigarette smoking initiation in the United States. Implications for smoking prevention policy. $\mathscr{F} A M A$ 1990;264:1550-5

23 Gilpin FA, Pierce JP. 1997. Trends in adolescent smoking initiation in the United States: is tobacco marketing an influence? Tobacco Control 1997;6:122-7.

24 Robertson SB, Jackson C. Initiation of cigarette smoking among children with and without learning disabilities. $\mathcal{F}$ Dev Behav Pediatr 1996;17:248-52.

25 Wolff CB, Portis M. Smoking, acculturation, and pregnancy outcome among Mexican Americans. Health Care for Women International 1997;17:563-73.

26 Klonoff EA, Landrine H. Acculturation and cigarette smoking among African American adults. F Behav Med 1996:19:501-14.

27 Dusenbery L, Epstein JA, Botvin GJ. The relationship between language spoken and smoking among HispanicLatino youth in New York City. Public Health Rep 1994;109:421-7.

28 Redfield R, Linton R, Herskovits MT. Memorandum for the study of acculturation. American Anthropologist 1936; 38:149-52.

29 Social Science Research Council Summer Seminar on Acculturation. Acculturation: an exploratory formulation. American Anthropologist 1954;56:973-1002.

30 Olmado EL. Acculturation: a psychological perspective. American Psychologist 1979;34:1061-70.

31 Cuellar I, Harris LC, Hasso R. An acculturation scale for Mexican American normal and clinical populations. Hexpanic f Behav Sci 1980;2:199-217.

32 Sodowsky GR, Lai EW, Plake BS. Moderating effects of social-cultural variables on acculturation attitudes of Hispanic and Asian Americans. F Counsel Dev 1991;70:194204.

33 Marin G, Perez-Stable EJ, Marin BV. Cigarette smoking among San Francisco Hispanics: the role of acculturation and gender. Am f Public Health 1989;79:196-8.

34 Deyo RA, Diehl AK, Hazuda $\mathrm{H}$, et al. A simple language-based acculturation scale for Mexican Americans: validation and application to health care research. Am $\mathcal{f}$ Public Health 1985;75:51-5.

35 Landrine H, Richardson JL, Klonoff EA, et al. Cultural diversity in the predictors of adolescent cigarette smoking: the relative influence of peers. F Behav Med 1994; the relative 
36 Sasao T. Statewide Asian Drug Service needs assessment: a multimethod approach. Sacramento, California: California Department of Alcohol and Drug Abuse, 1991.

37 Independent Evaluation Consortium. Final technical report: independent evaluation of the California Tobacco Control Prevention and Education Program: wave 1 data 1996-1997. Rockville, Maryland: Gallup Organization, 1998.

38 Popham WJ, Potter LD, Hetrik MA, et al. Effectiveness of the California 1990-1991 Tobacco Education Media Campaign. Am f Prev Med 1994;10:319-26.

39 Engels RC. Knibbe RA, Drop MJ. Inconsistencies in adolescents' self-reports of initiation of alcohol and tobacco use. Addict Behav 1997;22:613-23.

40 Cox DR, Oakes D. Analysis of survival data. London: Chapman \& Hall, 1984

41 Cox DR. Regression model and life-table (with discussion). F R Stat Soc Ser B 1972;34:187-220.

42 Chen X, Yin P, Peng J. Medical research design and dato analysis. Wuhan: Wuhan University Press, China, 1997.

43 Epstein JA, Botvin GJ, Dusenbury L, et al. Validation of an Epstein JA, Botvin GJ, Dusenbury L, et al. Validation of an Rep 1996;79:1075-9.

44 Mavreas V, Debbington P, Der G. The structure and validity of acculturation: analysis of an acculturation scale. Soc Psychiatry Psychiatr Epidemiol 1989;24:233-40.

45 Marmot MG, Syme SL. Acculturation and coronary heart disease in Japanese-Americans. F Epidemiol 1976;104:22547.

46 Gordon T. Mortality experience among the Japanese in the United States, Hawaii and Japan. Public Health Rep 1957; 72:543-53.

47 SAS Institute. The $S A S$ system for personal computers, release 6.12. Cary, North Carolina: SAS Institute, 1997.
48 Singer JD, Willett JB. Modeling the days of our lives: using survival analysis when designing and analyzing longitudisurvival analysis when designing and analyzing longitudi-
nal studies of duration and the timing of events. Psychol nal studies of duration

49 Douglas S, Hariharan G. The hazards of starting smoking: estimates from a split population duration model. 7 Health Econ 1994;13:213-30.

50 Flint AJ, Yamada EG, Novotny TE. Black-white differences in cigarette smoking uptake: progression from adolescent experimentation to regular use. Prev Med 1998;27:358-64.

51 Chen X. On East-West cultures and public health issues in China. Med Soc 1995;8:48-51.

52 Johnson CA. Pentz MA, Weber MD, et al. Relative effectiveness of comprehensive community programming for drug abuse prevention with high-risk and low-risk adolescents. $\mathcal{f}$ Consult Clin Psychol 1990;58:447-56.

53 Johnson CA, Farquhar JW, Sussman S. Methodological and substantive issues in substance abuse prevention research: an integration. Am Behav Sci 1996;39:935-42.

54 Dent CW, Sussman S, Ellickson P, et al. Is current drug abuse prevention programming generalizable across ethnic groups? Am Behav Sci 1996;39:911-18.

55 Graham JW, Johnson CA, Hansen WB, et al. Drug use prevention programs, gender, and ethnicity: evaluation of three seventh-grade project SMART cohorts. Prev Med 1990;19:305-13.

56 US Department of Health and Human Services. Strategies to control tobacco use in the United States: a blueprint for public health action in the 1990s. Bethesda, Maryland: Public Health Service, National Institutes of Health, National Cancer Institute, 1991. (NIH Publication No 92-3316.)

\section{Tobacco may be Bush's Achilles heel}

George W Bush $7 r$ is the clear favourite to win next year's presidential election, says Robert Dreyfuss, writing in The Nation. America's corporate elite are lining up behind him, his campaign coffers are full to overflowing, and he is way ahead in the polls. But he may have an Achilles heel: his close connections to the tobacco industry. For, as governor of Texas, Bush has been a good friend of "big tobacco". He resolutely opposed a federal lawsuit against the industry; he opposed increased taxes on cigarettes; and he has vigorously supported limits to the consumer's right to sue makers of dangerous products like tobacco. His close

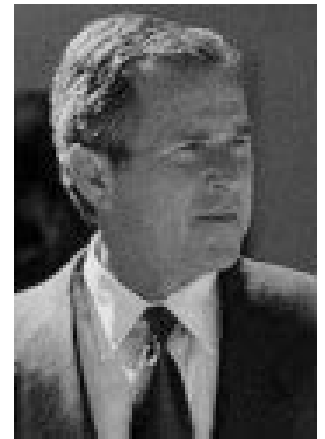
friend and long time chief political strategist, Karl Rove, was until 1996 formally on Philip Morris' payroll as a political intelligence operative. And tobacco has contributed handsomely to his $\$ 60$ million war chest. "The prospect of Bill Clinton gone and a George Bush presidency makes the industry almost giddy," says Martin Feldman, a tobacco industry analyst. Maybe so, but whether the health conscious American electorate will feel the same way remains to be seen. (Source: The Week, London, UK 20 November 1999, page 11) 